\title{
The nonexistence of robust codes for subsets of $\omega_{1}$
}

\author{
by
}

\author{
David Asperó (Bristol)
}

\begin{abstract}
Several results are presented concerning the existence or nonexistence, for a subset $S$ of $\omega_{1}$, of a real $r$ which works as a robust code for $S$ with respect to a given sequence $\left\langle S_{\alpha}: \alpha<\omega_{1}\right\rangle$ of pairwise disjoint stationary subsets of $\omega_{1}$, where "robustness" of $r$ as a code may either mean that $S \in L\left[r,\left\langle S_{\alpha}^{*}: \alpha<\omega_{1}\right\rangle\right]$ whenever each $S_{\alpha}^{*}$ is equal to $S_{\alpha}$ modulo nonstationary changes, or may have the weaker meaning that $S \in L\left[r,\left\langle S_{\alpha} \cap C: \alpha<\omega_{1}\right\rangle\right]$ for every club $C \subseteq \omega_{1}$. Variants of the above theme are also considered which result when the requirement that $S$ gets exactly coded is replaced by the weaker requirement that some set is coded which is equal to $S$ up to a club, and when sequences of stationary sets are replaced by decoding devices possibly carrying more information (like functions from $\omega_{1}$ into $\omega_{1}$ ).
\end{abstract}

1. Introduction. To this day, several ways have been found to code a given subset of $\omega_{1}$ in the presence of forcing axioms. All of the codings I am thinking of here are of the following form: One picks some object $\mathcal{D}$ in $H_{\omega_{2}}$ ( $\mathcal{D}$ will be the decoding device) in such a way that given any $A \subseteq \omega_{1}$ there is some $\delta<\omega_{2}$ and some $\omega_{1}$-club $\left(X_{i}\right)_{i<\omega_{1}}$ of $[\delta]^{\aleph_{0}}$ (or perhaps some real $r$ ) such that $A \in M$ whenever $M$ is an inner model such that $\left\{\mathcal{D},\left(X_{i}\right)_{i<\omega_{1}}\right\} \subseteq M$ (or $\{\mathcal{D}, r\} \subseteq M)$. This is illustrated by the following list.

THEOREM 1.1. Let $A$ be a subset of $\omega_{1}$.

(1) [J-So] If $\mathrm{MA}_{\omega_{1}}$ holds, then for every sequence $\left\langle r_{\alpha}: \alpha<\omega_{1}\right\rangle$ of pairwise almost disjoint infinite sets of integers there is a set of integers $r$ such that

$$
\alpha \in A \text { if and only if } r \cap r_{\alpha} \text { is infinite }
$$

for all $\alpha<\omega_{1}$.

(2) ([W, Theorem 5.14]) If Martin's Maximum (MM) holds, then for every stationary and co-stationary $T \subseteq \omega_{1}$ and every sequence $\left\langle S_{\alpha}: \alpha<\omega_{1}\right\rangle$ of pairwise disjoint stationary subsets of $\omega_{1}$ there is an ordinal $\delta<\omega_{2}$ and

2000 Mathematics Subject Classification: 03E47, 03E45, 03E50, 03E55, $03 \mathrm{E} 20$.

Key words and phrases: robust codes for subsets of $\omega_{1}$; sequences of stationary subsets of $\omega_{1}$; forcing axioms; $\mathbb{P}_{\max }$ extensions of $L(\mathbb{R})$. 
an $\subseteq$-increasing and $\subseteq$-continuous sequence $\left(X_{\nu}\right)_{\nu<\omega_{1}}$ of countable subsets of $\delta$ such that $\bigcup_{\nu} X_{\nu}=\delta$ and such that $\left(^{1}\right)$

$$
\operatorname{ot}\left(X_{\nu}\right) \in T \text { for every } \nu \in S_{\alpha} \text { whenever } \alpha \in A
$$

and

$$
\operatorname{ot}\left(X_{\nu}\right) \notin T \text { for every } \nu \in S_{\alpha} \text { whenever } \alpha \notin A \text {. }
$$

(3) ([W, Theorem 5.9]) If MM holds, then for all sequences $\left\langle S_{\alpha}: \alpha<\omega_{1}\right\rangle$ and $\left\langle T_{\alpha}: \alpha<\omega_{1}\right\rangle$ of pairwise disjoint stationary subsets of $\omega_{1}$ there is an ordinal $\delta<\omega_{2}$ of cofinality $\omega_{1}$ and there is a strictly increasing and continuous $f: \omega_{1} \rightarrow \delta$ cofinal in $\delta$ such that $f$ " $T_{\alpha} \subseteq \widetilde{S}_{h(\alpha)}$ for all $\alpha<\omega_{1}$, where $h: \omega_{1} \rightarrow \omega_{1}$ is the enumerating function of $A$, and $\widetilde{B}$ is, given a set $B \subseteq \omega_{1}$, the set of all $\gamma$ between $\omega_{1}$ and $\omega_{2}$ such that, given any surjection $\pi: \omega_{1} \rightarrow \gamma$, there is a club $C \subseteq \omega_{1}$ such that ot $\left(\pi^{\prime \prime} \nu\right) \in B$ for all $\nu \in C$ (equivalently, such that $\Vdash_{\mathcal{P}\left(\omega_{1}\right) / \mathrm{NS}_{\omega_{1}}} \gamma \in j(B)$, where $\mathrm{NS}_{\omega_{1}}$ is the nonstationary ideal on $\omega_{1}$ and $j$ denotes the generic elementary embedding obtained from forcing with $\left.\mathcal{P}\left(\omega_{1}\right) / \mathrm{NS}_{\omega_{1}}\left({ }^{2}\right)\right)$.

(4) ([T]) If Bounded Martin's Maximum (BMM) holds, then for every sequence $\left\langle r_{\alpha}: \alpha<\omega_{1}\right\rangle$ of pairwise distinct members of $2^{\omega}$ and every sequence $\left\langle S_{\alpha}: \alpha<\omega_{1}\right\rangle$ of pairwise disjoint stationary subsets of $\omega_{1}$ there are ordinals $\beta<\gamma<\delta<\omega_{2}$ and an $\subseteq$-increasing and $\subseteq$-continuous sequence $\left(X_{\nu}\right)_{\nu<\omega_{1}}$ of countable subsets of $\delta$ such that $\bigcup_{\nu} X_{\nu}=\delta$ and

(a) $\max \Delta\left(r_{\mathrm{ot}\left(X_{\nu} \cap \beta\right)}, r_{\mathrm{ot}\left(X_{\nu} \cap \gamma\right)}, r_{\mathrm{ot}\left(X_{\nu}\right)}\right)=\Delta\left(r_{\mathrm{ot}\left(X_{\nu} \cap \beta\right)}, r_{\mathrm{ot}\left(X_{\nu} \cap \gamma\right)}\right)$ for all $\nu \in S_{\alpha}$ whenever $\alpha \in A$,

(b) $\min \Delta\left(r_{\mathrm{ot}\left(X_{\nu} \cap \beta\right)}, r_{\mathrm{ot}\left(X_{\nu} \cap \gamma\right)}, r_{\mathrm{ot}\left(X_{\nu}\right)}\right)=\Delta\left(r_{\mathrm{ot}\left(X_{\nu} \cap \beta\right)}, r_{\mathrm{ot}\left(X_{\nu} \cap \gamma\right)}\right)$ for all $\nu \in S_{\alpha}$ whenever $\alpha \notin A$,

where $\Delta\left(r_{0}, r_{1}\right)=\min \left\{m: r_{0}(m) \neq r_{1}(m)\right\}$ for all distinct reals $r_{0}, r_{1}$, and $\Delta\left(r_{0}, r_{1}, r_{2}\right)$ is the two-element set $\left\{\Delta\left(r_{0}, r_{1}\right), \Delta\left(r_{0}, r_{2}\right), \Delta\left(r_{1}, r_{2}\right)\right\}$ for all distinct reals $r_{0}, r_{1}, r_{2} \in 2^{\omega}$.

(5) $([\mathrm{M}])$ If the Bounded Proper Forcing Axiom holds, then given any ladder system $\left\langle C_{\xi}: \xi \in \operatorname{Lim}\left(\omega_{1}\right)\right\rangle\left({ }^{3}\right)$ and any sequence $\left\langle S_{\alpha}: \alpha<\omega_{1}\right\rangle$ of pairwise disjoint stationary subsets of $\omega_{1}$ there is an ordinal $\delta<\omega_{2}$ and an $\subseteq$-increasing and $\subseteq$-continuous sequence $\left(X_{\nu}\right)_{\nu<\omega_{1}}$ of countable subsets of $\delta$ such that $\bigcup_{\nu} X_{\nu}=\delta$ and such that for every limit $\nu<\omega_{1}$ there is $\nu_{0}<\nu$

$\left({ }^{1}\right)$ Given a set of ordinals $X$, ot $(X)$ will denote the order type of $X$.

$\left(^{2}\right)$ Given an ordinal $\gamma<\omega_{2}$ and a surjection $\pi: \omega_{1} \rightarrow \gamma$, the function on $\omega_{1}$ sending every $\nu$ to ot $\left(\pi^{\prime \prime} \nu\right)$ represents, in the generic ultrapower derived from forcing with $\mathcal{P}\left(\omega_{1}\right) / \mathrm{NS}_{\omega_{1}}$, an ordinal of order type $\gamma$. We call such a function a canonical function for $\gamma$.

$\left({ }^{3}\right)$ That is, each $C_{\xi}$ is a subset of $\xi$ of order type $\omega$ and with supremum $\xi$. 
such that for all $\xi, \nu_{0}<\xi<\nu$,

$$
X_{\nu} \cap \omega_{1} \in \bigcup_{\alpha \in A} S_{\alpha} \text { if and only if } w\left(X_{\xi} \cap \omega_{1}, X_{\nu} \cap \omega_{1}\right)<w\left(X_{\xi}, X_{\nu}\right)
$$

where, given two sets $X \subseteq Y$ of countable ordinals, $w(X, Y)$ denotes the cardinality of $\sup (X) \cap \pi_{Y}^{-1}$ " $C_{\mathrm{ot}(Y)}$ (and $\pi_{Y}$ is the transitive collapse of $Y$ ).

A well known theorem of H. Woodin ([W, Theorems 3.16, 3.17]) says that if $\mathrm{NS}_{\omega_{1}}$ is saturated and $\mathcal{P}\left(\omega_{1}\right)^{\sharp}$ exists, then every club of $\omega_{1}$ contains a club which is constructible from a real, and thus the second uniform indiscernible $u_{2}$ is $\omega_{2}$ (and, as a consequence, for every ordinal $\delta<\omega_{2}$ there is a real $r$ such that $|\delta|^{L[r]}$ is at most the $\omega_{1}$ of $\left.V\right)$. On the other hand, by a result of R. Schindler $[S]$, the existence of the sharp of every set of ordinals follows already from BMM. Hence, (1), (4) and (5) from Theorem 1.1 are instances of the following fact (since the relevant extra decoding objects, like the fixed ladder system in (5), can certainly be coded by a stationary and costationary subset of $\left.\omega_{1}\right)$ :

- If MM holds (more generally, if BMM holds and $\mathrm{NS}_{\omega_{1}}$ is saturated), then there is a sequence $\left\langle S_{\alpha}: \alpha<\omega_{1}\right\rangle$ of pairwise disjoint stationary subsets of $\omega_{1}$ such that given any subset $A$ of $\omega_{1}$ there is a real $r$ with $A \in L\left[r,\left\langle S_{\alpha}: \alpha<\omega_{1}\right\rangle\right]$.

Likewise, (2) and (3) from Theorem 1.1 take obviously the following stronger general form:

- If MM holds $\left(^{4}\right)$, then given any sequence $\left\langle S_{\alpha}: \alpha<\omega_{1}\right\rangle$ of pairwise disjoint stationary subsets of $\omega_{1}$ and any subset $A$ of $\omega_{1}$ there is a real $r$ such that $A \in L\left[r,\left\langle S_{\alpha}: \alpha<\omega_{1}\right\rangle\right]$.

Thus, sufficiently strong forcing axioms imply the existence, given an arbitrary subset $A$ of $\omega_{1}$ and an arbitrary sequence of length $\omega_{1}$ of mutually disjoint stationary subsets of $\omega_{1}$, of a real which works as a code for $A$ with respect to our sequence of stationary sets. This is also true, by the same considerations, about Woodin's $\mathbb{P}_{\max }$ axiom $(*)\left({ }^{5}\right)$ : $\operatorname{Both}(2)$ and $(3)$ in Theorem 1.1 are true under $(*)$ (by [W], Lemma 5.18, and [W, Lemma 5.5], respectively), and not in a vacuous way (that is, $(*)$ does imply the existence of $\omega_{1}$-sequences of mutually disjoint stationary subsets of $\left.\omega_{1}\left({ }^{6}\right)\right)$, and so is the fact that every club of $\omega_{1}$ contains a club constructible from a real $\left({ }^{7}\right)$.

$\left({ }^{4}\right)$ For (2) already BMM plus the saturation of $\mathrm{NS}_{\omega_{1}}$ suffice.

$\left({ }^{5}\right)$ Namely that $\mathrm{AD}$ holds in $L(\mathbb{R})$ and that $L\left(\mathcal{P}\left(\omega_{1}\right)\right)$ is a $\mathbb{P}_{\max }$ extension of $L(\mathbb{R})$ (for the definition of $\mathbb{P}_{\max }$ forcing and its basic theory the reader is referred to Chapter 4 of $[\mathrm{W}]) ; \mathbb{P}_{\max }$ is a partial order in $L(\mathbb{R})$, which, under $\mathrm{AD}^{L(\mathbb{R})}$, forces over $L(\mathbb{R})$ the Axiom of Choice and that $(*)$ holds (by [W, Lemma 4.38, Theorem 4.50 and Lemma 5.5]).

$\left({ }^{6}\right)$ By [W, Theorem 4.50].

$\left({ }^{7}\right)$ Again by [W, Theorem 4.50]. 
Although in this paper I will focus mainly on decoding devices consisting of an $\omega_{1}$-sequence of mutually disjoint stationary subsets of $\omega_{1}$, it is worth remarking at this point that the simplest possible decoding device one may think of - namely a single subset of $\omega_{1}$ which is both stationary and costationary - does do its job under strong enough forcing axioms or under $(*)$ :

OBSERVATION 1.1. Suppose that BMM holds and $\mathrm{NS}_{\omega_{1}}$ is saturated, or else that the $\mathbb{P}_{\max }$ axiom $(*)$ holds. Then, given any stationary and costationary $S \subseteq \omega_{1}$ and any $A \subseteq \omega_{1}$ there is a real $r$ such that $A \in L[r, S]$.

Proof. Under the first hypothesis, this is a consequence of the following corollary to a result due, independently, to P. Larson and H. Woodin (see the proof of Theorem 5.14 in [W]):

FACT 1.1. If $\mathrm{NS}_{\omega_{1}}$ is saturated, then the set of $X \in\left[\omega_{2}\right]^{\aleph_{0}}$ such that $X \cap \omega_{1} \in S$ and such that $\operatorname{ot}(X) \in T$ if and only if $X \cap \omega_{1} \in A$ is a stationary subset of $\left[\omega_{2}\right]^{\aleph_{0}}$ whenever $S, T$ and $\omega_{1} \backslash T$ are stationary subsets of $\omega_{1}$ and $A$ is a subset of $\omega_{1}$.

Fix a sequence $\left\langle r_{\alpha}: \alpha<\omega_{1}\right\rangle$, where each $r_{\alpha}$ is a set of integers coding $\chi_{A}\left\lceil\alpha\right.$ and $\chi_{A}: \omega_{1} \rightarrow\{0,1\}$ is the characteristic function of $A$. Fix also a partition $\left\langle S_{\alpha}: \alpha<\omega_{1}\right\rangle$ of $\omega_{1}$ into stationary sets. Pick any $n<\omega$. By Fact 1.1, the standard forcing notion $\mathcal{P}$ for shooting, with countable conditions, an $\omega_{1}$-club inside $\left\{X \in\left[\omega_{2}\right]^{\aleph_{0}}:\right.$ ot $(X) \in S$ iff $X \cap \omega_{1} \in \bigcup\left\{S_{\alpha}\right.$ : $\left.\left.\alpha<\omega_{1}, n \in r_{\alpha}\right\}\right\}\left(^{8}\right)$ preserves stationary subsets of $\omega_{1}$. Hence, by BMM there is some $\alpha_{n}<\omega_{2}$ such that, given any surjection $\pi: \omega_{1} \rightarrow \alpha_{n}$, there is a club $C \subseteq \omega_{1}$ such that

$$
\{\nu \in C: \operatorname{ot}(\pi \cdot \nu) \in S\}=C \cap \bigcup\left\{S_{\alpha}: \alpha<\omega_{1}, n \in r_{\alpha}\right\} .
$$

Since $\mathrm{NS}_{\omega_{1}}$ is saturated and the sharp of every set exists, every club of $\omega_{1}$ contains a club constructible from a real and $u_{2}$ is $\omega_{2}$. Therefore we may find a real $r$ coding the sequence $\left(\alpha_{n}\right)_{n}$ as well as clubs witnessing the above equality for surjections from $\omega_{1}^{V}$ to the $\alpha_{n}$ 's which are constructible from reals. Thus, we may assume that, for some club $C$ of $\omega_{1}$ in $L[r, S]$, the sequence indexed by $n<\omega$ of the pairs

$$
\left\langle\bigcup\left\{S_{\alpha} \cap C: \alpha<\omega_{1}, n \in r_{\alpha}\right\}, \bigcup\left\{S_{\alpha} \cap C: \alpha<\omega_{1}, n \notin r_{\alpha}\right\}\right\rangle
$$

belongs to $L[r, S]$. Now pick any $\xi \in C$ and let $s_{\xi}$ be the set of integers $n$ such that $\xi$ is in the first component of the $n$th member of the above $\omega$-sequence, that is, such that $\xi$ is in $\bigcup\left\{S_{\alpha} \cap C: \alpha<\omega_{1}, n \in r_{\alpha}\right\}$. Then $\left\langle s_{\xi}: \xi \in C\right\rangle$ is clearly constructible from $r$ together with $S$ and, given $\xi \in C$ and $n<\omega$, we have $n \in s_{\xi}$ exactly when $n \in r_{\alpha}$, where $\alpha<\omega_{1}$ is unique

$\left({ }^{8}\right) \mathcal{P}$ consists of initial segments of such a club of length a successor countable ordinal ordered by extension. 
such that $\xi \in S_{\alpha}$. It follows that the unordered set $Y=\left\{r_{\alpha}: \alpha<\omega_{1}\right\}$, being equal to $\left\{s_{\xi}: \xi \in C\right\}$, belongs to $L[r, S]$. Finally, consider the map on $Y$ sending every $s_{\xi}$ to the unique ordinal $\alpha$ such that $s_{\xi}$ codes a function from $\alpha$ into $\{0,1\}$. This function, which can be read off from $Y$, sends $s_{\xi}$ to $\alpha$ exactly when $\xi$ is in $S_{\alpha}$. Therefore the sequence $\left\langle r_{\alpha}: \alpha<\omega_{1}\right\rangle$ belongs to $L[r, S]$, and so in particular does $A$.

Under the assumption that $(*)$ holds this is proved exactly in the same way using Woodin's results on $\mathbb{P}_{\max }$ mentioned before.

All codings expressed in Theorem 1.1 may be sensitive to nonstationary changes in the decoding device $\left\langle S_{\alpha}: \alpha<\omega_{1}\right\rangle$. In (2), for instance, the fact that $S_{\alpha} \Vdash_{\mathcal{P}\left(\omega_{1}\right) / \mathrm{NS}_{\omega_{1}}} \delta \in j(T)$ need not imply that $S_{\alpha} \Vdash_{\mathcal{P}\left(\omega_{1}\right) / \mathrm{NS}_{\omega_{1}}} \delta \in j\left(T^{*}\right)$ if $T^{*} \subseteq \omega_{1}$ is such that the symmetric difference $T \triangle T^{*}$ is nonstationary. However, in the present context it seems natural to ask for the existence of robust codings for subsets of $\omega_{1}$, where "robustness" of a real as a code for a given set $A \subseteq \omega_{1}$ with respect to a sequence $\left\langle S_{\alpha}: \alpha<\omega_{1}\right\rangle$ of pairwise disjoint stationary sets means that the fact that $A$ gets coded by $r$ and $\left\langle S_{\alpha}: \alpha<\omega_{1}\right\rangle$, that is, that

$$
A \in L\left[r,\left\langle S_{\alpha}: \alpha<\omega_{1}\right\rangle\right]
$$

does not depend on any alteration of the members of the decoding device $\left\langle S_{\alpha}: \alpha<\omega_{1}\right\rangle$ by a nonstationary set, but only on the class of the $S_{\alpha}$ 's in $\mathcal{P}\left(\omega_{1}\right) / \mathrm{NS}_{\omega_{1}}\left({ }^{9}\right)$. This question, as well as variants of it resulting from changing the meaning of the expressions "robust" and "coding" in some slight way (and from considering decoding devices in $H_{\omega_{2}}$ possibly more powerful than the above sequences), are the questions being addressed in Sections 2 and 3.

Acknowledgements. I thank an anonymous referee for finding a simplified proof of an earlier version of Corollary 3.1 in Section 3 (and thereby weakening the hypothesis used there), and for allowing me to incorporate this version into the paper.

2. Slightly perturbing a decoding device. Theorem 2.1 below shows, under the assumption that the sharp of every real exists, that robust codes - in the sense of the previous section - for stationary and co-stationary stationary subsets of $\omega_{1}$ simply do not exist.

A few words on the history of Theorem 2.1 may be in order here. In [L] Larson forced, over a model with a supercompact limit of supercompact cardinals, to obtain a model in which (a strong form of) MM holds and (*)

$\left({ }^{9}\right)$ The codings expressed in (2) and (3) certainly are independent of changes in the $S_{\alpha}$ 's modulo countable sets, and the decoding devices used in (1), (4) and (5) can of course be chosen so that this is also the case with them. 
fails. I observed that in Larson's model there is an $\omega_{1}$-sequence of stationary subsets of $\omega_{1}$ which admits no robust code with respect to any $\omega_{1}$-sequence of mutually disjoint stationary subsets of $\omega_{1}$. This was the starting point of the present paper. Eventually I proved, using some theory of $\mathbb{P}_{\max }$ forcing, that a relatively strong large cardinal assumption $\left({ }^{10}\right)$ implies, in the universe, the nonexistence of robust codes for any stationary and co-stationary subset of $\omega_{1}$. Then Woodin provided a different argument for deriving the same conclusion from just the existence of a Woodin cardinal below a measurable. Moreover, in his proof the conclusion applied actually to any subset of $\omega_{1}$ not constructible from any real. I noticed that essentially the same argument could be applied just assuming that $x^{\dagger}$ exists for every real $x$, and finally Woodin pointed out that the same kind of argument should work in the context of just the existence of sharps for reals. This, of course, is the optimal hypothesis for dealing with stationary and co-stationary subsets of $\omega_{1}$ (Corollary 2.1).

Theorem 2.1 (Woodin). Assume $x^{\sharp}$ exists for every real $x$. Then, given any $A \subseteq \omega_{1}$, any sequence $\left\langle S_{\alpha}: \alpha<\omega_{1}\right\rangle$ of pairwise disjoint subsets of $\omega_{1}$ and any real $r$, if $A$ is not constructible from a real, then there are sets $S_{\alpha}^{*}$ for $\alpha<\omega_{1}$ such that $S_{\alpha} \triangle S_{\alpha}^{*}$ is nonstationary for all $\alpha$, and yet $A \notin L\left[r,\left\langle S_{\alpha}^{*}: \alpha<\omega_{1}\right\rangle\right]$.

Proof. Assume that the sharp of every real exists and suppose that $A$, $\left\langle S_{\alpha}: \alpha<\omega_{1}\right\rangle$ and $r$ witness the failure of the theorem. By enlarging $S_{0}$ and shrinking the $S_{\alpha}$ 's for $\alpha \neq 0$ slightly, adding one member to the above sequence, and/or switching the indices of two of its members if necessary, we may assume that $S_{\alpha} \cap(\alpha+1)$ is empty for all $\alpha>1$ and that $\left\{S_{\alpha}: \alpha<\omega_{1}\right\}$ is a partition of $\omega_{1}$. We shall see that $A$ is constructible from a real, which will be a contradiction. Let $\left\langle\gamma_{\xi}: \xi \in\right.$ Ord $\rangle$ be the increasing enumeration of the class of Silver indiscernibles for $L[r]$. Given any ordinal $\gamma$ let $\mathcal{P}_{<\gamma}$ be the partial order, ordered by extension, consisting of all finite functions $p \subseteq \gamma \times \gamma$ such that $p(\nu)<\nu$ for every $\nu$ in the domain of $p$. We will construct a certain sequence $\left\langle g_{\xi}: \xi<\omega_{1}\right\rangle$ such that

(a) each $g_{\xi}$ is generic for $\mathcal{P}_{<\gamma_{\xi}}$ over $L[r]$,

(b) $g_{\xi}=g_{\xi^{\prime}} \cap \mathcal{P}_{<\gamma_{\xi}}$ for all $\xi<\xi^{\prime}<\omega_{1}$,

(c) $G:=\bigcup_{\xi<\omega_{1}} g_{\xi}$ is generic for $\mathcal{P}_{<\omega_{1}^{V}}$ over $L[r]$.

Since each $\mathcal{P}\left(\gamma_{\xi}\right)^{L[r]}$ is countable, the construction above can be carried out. Also, (c) follows automatically from (a) and (b) since $\mathcal{P}_{<\omega_{1}^{V}}$ has the $\omega_{1}^{V}$ chain condition in $L[r]$. This sequence will be built by recursion on $\xi<\omega_{1}$

$\left({ }^{10}\right)$ Specifically, the assumption that $\mathrm{AD}^{L(\mathbb{R})}$ holds, together with the existence of a Woodin cardinal below a measurable and with the invariance of the theory of $L(\mathbb{R})$ under small ccc forcing. 
using some fixed surjection $\sigma: \omega_{1} \rightarrow \omega_{1} \times \omega$ with the property that $\sigma(\xi)=$ $\langle\alpha, n\rangle$ only if $\alpha \leq \xi$. For each $\xi<\omega_{1},\left\langle b_{n}^{\xi}: n<\omega\right\rangle$ will be some enumeration of all subsets of $\gamma_{\xi}$ in $L[r]\left[g_{\xi}\right]$.

Note that, given any $\xi<\xi^{\prime}<\omega_{1}$ and any filters $g$ and $g^{\prime}$, generic for $\mathcal{P}_{<\gamma_{\xi}}$ and $\mathcal{P}_{<\gamma_{\xi^{\prime}}}$, respectively, satisfying the coherence property expressed in (b), the map sending the interpretation in $L[r]$ of a Skolem term applied to a strictly increasing sequence

$$
\left\langle\gamma_{\xi_{0}}, \ldots, \gamma_{\xi_{n}}, \gamma_{\xi}, \gamma_{\xi+i_{0}}, \ldots, \gamma_{\xi+i_{m}}\right\rangle
$$

of Silver indiscernibles for $L[r]$ to the interpretation of the same term applied to the sequence

$$
\left\langle\gamma_{\xi_{0}}, \ldots, \gamma_{\xi_{n}}, \gamma_{\xi^{\prime}}, \gamma_{\xi^{\prime}+i_{0}}, \ldots, \gamma_{\xi^{\prime}+i_{m}}\right\rangle
$$

extends to a unique elementary embedding from $L[r][g]$ into $L[r]\left[g^{\prime}\right]$. We shall denote this embedding by $j_{g, g^{\prime}}$. It follows that if $\left\langle g_{\xi}: \xi<\omega_{1}\right\rangle$ satisfies (a) and (b) above, then $\left\langle L\left[r^{\prime}\right]\left[g_{\xi}\right], j_{g_{\xi}, g_{\xi^{\prime}}}: \xi \leq \xi^{\prime}<\omega_{1}\right\rangle$ is a directed system. Then we let $j_{g_{\xi}, G}: L[r]\left[g_{\xi}\right] \rightarrow L[r][G]$ denote, for any given $\xi$, the limit map from $L[r]\left[g_{\xi}\right]$ into the limit object $L[r][G]\left(G\right.$ will obviously be $\left.\bigcup_{i<\omega_{1}} g_{i}\right)$ corresponding to this directed system.

Pick $g_{0}$ arbitrarily and let $\left\langle s_{\beta}: \beta<\gamma_{0}\right\rangle$ be the sequence in $L[r]\left[g_{0}\right]$ consisting of pairwise disjoint stationary subsets of $\gamma_{0}\left(=\omega_{1}^{L[r]\left[g_{0}\right]}\right)$ defined by setting $s_{\beta}=\left(\bigcup g_{0}\right)^{-1}(\beta)$ for every $\beta<\gamma_{0}$. Now pick $\xi<\omega_{1}$ and suppose $g_{\xi}$ has been built. Then choose $g_{\xi+1}$ in such a way that the following two conditions are met:

(1) If $\alpha<\omega_{1}$ is such that $\gamma_{\xi} \in S_{\alpha}$, then $\gamma_{\xi} \in j_{g_{0}, g_{\xi+1}}\left(\left\langle s_{\beta}: \beta<\gamma_{0}\right\rangle\right)(\alpha)$.

(2) If there is some generic filter $g$ for $\mathcal{P}_{<\gamma_{\xi+1}}$ over $L[r]$ such that $g \cap$ $\mathcal{P}_{<\gamma_{\xi}}=g_{\xi}$, such that $j_{g_{0}, g}$ satisfies the requirement expressed in (1) and, moreover, such that $j_{g_{\alpha}, g}\left(b_{n}^{\alpha}\right) \neq A \cap \gamma_{\xi+1}($ where $\sigma(\xi)=\langle\alpha, n\rangle)$, then we let $g_{\xi+1}$ be such a filter.

These conditions can obviously be satisfied by the definition of $\left\langle s_{\beta}\right.$ : $\left.\beta<\gamma_{0}\right\rangle$ in $L[r]\left[g_{0}\right]$. Let $S_{\alpha}^{*}$ be, for each $\alpha, j_{g_{0}, G}\left(\left\langle s_{\beta}: \beta<\gamma_{0}\right\rangle\right)(\alpha)$. Clearly, for each $\xi$ and each $\alpha<\omega_{1}, \gamma_{\xi} \in S_{\alpha}^{*}$ if and only if $\gamma_{\xi} \in S_{\alpha}$. Now suppose $A \in L\left[r,\left\langle S_{\alpha}^{*}: \alpha<\omega_{1}\right\rangle\right]$. Then $A=j_{g_{\alpha}, G}(b)$ for some subset $b$ of $\gamma_{\alpha}$ belonging to $L[r]\left[g_{\alpha}\right]$ and some $\alpha$. Let $n$ be such that $b=b_{n}^{\alpha}$, let $\xi \geq \alpha$ be such that $\sigma(\xi)=\langle\alpha, n\rangle$, and let $\beta<\omega_{1}$ be such that $\gamma_{\xi} \in S_{\beta}$. The theorem will be proved once we show that $A$ is in $L[a]$ whenever $a$ is a real coding $r$ and $g_{\xi}$.

Take such an $a$ and consider, in $L[a]$, the set $B$ consisting of all those ordinals $\gamma<\omega_{1}^{V}$ for which there is some condition $p$ in $\mathcal{P}_{<\omega_{1}}$ such that $p \cap \mathcal{P}_{<\gamma_{\xi}} \in g_{\xi}, p\left(\gamma_{\xi}\right)=\beta$, and such that $p\left\lceil\left[\gamma_{\xi}, \omega_{1}\right)\right.$ forces in $\left.\mathcal{P}_{\left[\gamma_{\xi}, \omega_{1}\right)}{ }^{11}\right)$

$\left({ }^{11}\right)$ This notation obviously represents the suborder of $\mathcal{P}_{<\omega_{1}}$ consisting of those functions with domain disjoint from $\gamma_{\xi}$. 
over $L[r]\left[g_{\xi}\right]$ that $\gamma \in j_{g_{\alpha}, \dot{G}}(b)$, where $\dot{G}$ is a name for the generic object for $\mathcal{P}_{<\omega_{1}^{V}}$ which is the union of $g_{\xi}$ and the generic object for $\mathcal{P}_{\left[\gamma_{\xi}, \omega_{1}\right)}$. It suffices to see that $B$ is $A$. Suppose there is some $\gamma \in A \backslash B$ (the case that $\gamma$ is in $B \backslash A$ is argued for symmetrically). Then there is a condition $p$ in $\mathcal{P}_{<\omega_{1}}$ compatible with $g_{\xi}$ which sends $\gamma_{\xi}$ to $\beta$ and such that $p\left\lceil\left[\gamma_{\xi}, \omega_{1}\right)\right.$ forces in $\mathcal{P}_{\left[\gamma_{\xi}, \omega_{1}\right)}$ over $L[r]\left[g_{\xi}\right]$ that $\gamma$ is not in $j_{g_{\alpha}, \dot{G}}(b)$. As $\gamma$ is in $A\left(=j_{g_{\alpha}, G}(b)\right)$, there is also a condition $p^{\prime}$ in $G$ sending $\gamma_{\xi}$ to $\beta$ and forcing that $\gamma$ is in $j_{g_{\alpha}, \dot{G}}(b)$. Now, by indiscernibility, there is an ordinal $\gamma^{*}<\gamma_{\xi+1}$ and there are conditions $q$ and $q^{\prime}$ in $\mathcal{P}_{<\gamma_{\xi+1}}$ sending $\gamma_{\xi}$ to $\beta$ and such that $q \cap \mathcal{P}_{<\gamma_{\xi}}$ and $q^{\prime} \cap \mathcal{P}_{<\gamma_{\xi}}$ are both in $g_{\xi}$ and $q\left\lceil\left[\gamma_{\xi}, \gamma_{\xi+1}\right)\right.$ forces in $\mathcal{P}_{\left[\gamma_{\xi}, \gamma_{\xi+1}\right)}$ over $L[r]$ that $\gamma^{*}$ is not in $j_{g_{\alpha}, \dot{g}_{\xi+1}}(b)\left({ }^{12}\right)$, whereas $q^{\prime} \uparrow\left[\gamma_{\xi}, \gamma_{\xi+1}\right)$ forces that $\gamma^{*}$ is in $j_{g_{\alpha}, \dot{g}_{\xi+1}}(\dot{b})$. It follows that we have been able to choose $g_{\xi+1}$ in such a way that $\gamma^{*} \notin j_{g_{\alpha}, G}(b)$ if $\gamma^{*} \in A$ and $\gamma^{*} \in j_{g_{\alpha}, G}(b)$ if $\gamma^{*} \notin A$, and this contradicts the fact that $j_{g_{\alpha}, G}(b)$ was supposed to be $A$.

Next I will consider the situation in which we are given a set $A \subseteq \omega_{1}$ and a real $r$ which is meant to be a code for (an approximation of) $A$ with respect to a sequence $\mathcal{S}=\left\langle S_{\alpha}: \alpha<\omega_{1}\right\rangle$ of stationary sets, and we look at the weaker version of robustness for $r$ arising when we just ask that the class of $A$ in $\mathcal{P}\left(\omega_{1}\right) / \mathrm{NS}_{\omega_{1}}$ (rather than $A$ itself) be coded; this is the version of robustness, that is, that results when the demand that $A$ itself be constructible from $r$ together with any decoding device $\mathcal{S}^{*}$ almost equal to $\mathcal{S}$ is weakened to the requirement that, for every such $\mathcal{S}^{*}$, there is some set in $L\left[r, \mathcal{S}^{*}\right]$ which is equal to $A$ up to a club. This kind of variant of the original situation will be taken up again in the next section. Note that if we are to obtain a negative result along the lines of Theorem 2.1, some restriction on $A$ and on the $S_{\alpha}$ 's will have to be taken into account: If $A$ is (almost equal to) $\bigcup_{\alpha \in X} S_{\alpha}$ for some $X \in L$, then any small variation on the $S_{\alpha}$ 's will construct a set almost equal to $A$.

Theorem 2.2. Assume $x^{\sharp}$ exists for every real $x$. Then, given any $S \subseteq$ $\omega_{1}$, any sequence $\left\langle S_{\alpha}: \alpha<\omega_{1}\right\rangle$ of mutually disjoint subsets of $\omega_{1}$ and any real $r$, if there is some $\alpha_{0}$ such that $S \cap S_{\alpha_{0}}$ and $S_{\alpha_{0}} \backslash S$ are both stationary, then there are sets $S_{\alpha}^{*}\left(\alpha<\omega_{1}\right)$ such that

(a) $S_{\alpha} \triangle S_{\alpha}^{*}$ is nonstationary for all $\alpha$,

(b) $S^{*} \notin L\left[r,\left\langle S_{\alpha}^{*}: \alpha<\omega_{1}\right\rangle\right]$ whenever $S \triangle S^{*}$ is nonstationary.

Proof. Suppose the theorem fails. Again we may assume that $S_{\alpha} \cap$ $(\alpha+1)$ is empty for all $\alpha>1$ and that $\bigcup_{\alpha} S_{\alpha}=\omega_{1}$. Fix a sequence

$\left({ }^{12}\right)$ Again $\mathcal{P}_{\left[\gamma_{\xi}, \gamma_{\xi+1}\right)}$ has the natural meaning and $\dot{g}_{\xi+1}$ is a $\mathcal{P}_{\left[\gamma_{\xi}, \gamma_{\xi+1}\right)}$-term for the generic filter for $\mathcal{P}_{<\gamma_{\xi+1}}$ which is the union of $g_{\xi}$ and the generic filter for $\mathcal{P}_{\left[\gamma_{\xi}, \gamma_{\xi+1}\right)}$. 
$\left\langle T_{n}^{\alpha}: \alpha<\omega_{1}, n<\omega\right\rangle$ of mutually disjoint stationary subsets of $S_{\alpha_{0}}$ with the property that all $T_{n}^{\alpha} \cap S$ and all $T_{n}^{\alpha} \backslash S$ are stationary.

Letting all undefined notions here stand for corresponding notions in the construction in the proof of Theorem 2.1, this time we build a sequence $\left\langle g_{\xi}: \xi<\omega_{1}\right\rangle$ satisfying properties (a)-(c) in that proof in such a way that, given any $\xi<\omega_{1}$ for which $g_{\xi}$ has already been built, $g_{\xi+1}$ is such that the requirement corresponding to (1) in the proof of Theorem 2.1 is met and, if possible, such that $\gamma_{\xi} \in j_{g_{\alpha}, g_{\xi+1}}\left(d_{n}^{\alpha}\right)$ if $\gamma_{\xi} \in T_{n}^{\alpha}$ and $\alpha_{0}<\alpha \leq \xi$ (where, given any $\alpha,\left\langle d_{n}^{\alpha}: n\langle\omega\rangle\right.$ is now some fixed enumeration of all subsets of $j_{g_{0}, g_{\alpha}}\left(\left\langle s_{\beta}: \beta<\gamma_{0}\right\rangle\right)\left(\alpha_{0}\right)$ in $\left.L[r]\left[g_{\alpha}\right]\right)$.

Again we let $S_{\alpha}^{*}$ be, for each $\alpha, j_{g_{0}, G}\left(\left\langle s_{\beta}: \beta<\gamma_{0}\right\rangle\right)(\alpha)$. As in Theorem 2.1, for all $\xi$ and $\alpha<\omega_{1}, \gamma_{\xi} \in S_{\alpha}$ if and only if $\gamma_{\xi} \in S_{\alpha}^{*}$. Now suppose $S^{*}$ is some set in $L\left[r,\left\langle S_{\alpha}^{*}: \alpha<\omega_{1}\right\rangle\right]$ almost equal, modulo $\mathrm{NS}_{\omega_{1}}$, to $S$. Then $S^{*} \cap S_{\alpha_{0}}^{*}$ has to be stationary. Let $n<\omega$ and $\alpha_{0}<\alpha<\omega_{1}$ be such that $S^{*} \cap S_{\alpha_{0}}^{*}=j_{g_{\alpha}, G}\left(d_{n}^{\alpha}\right)$, and let $\xi<\xi^{\prime}$ be arbitrarily chosen so that $\alpha<\xi, \gamma_{\xi} \in T_{n}^{\alpha}$, and $\gamma_{\xi^{\prime}} \in S^{*} \cap S_{\alpha_{0}}^{*}$. Then, since $\gamma_{\xi}$ and $\gamma_{\xi^{\prime}}$ are Silver indiscernibles for $L[r]$ and since $S^{*} \cap S_{\alpha_{0}}^{*} \cap \gamma_{\xi^{\prime}+1}=j_{g_{\alpha}}, g_{\xi^{\prime}+1}\left(d_{n}^{\alpha}\right)$, there is a condition $p$ in $\mathcal{P}_{\left[\gamma_{\xi}, \gamma_{\xi+1}\right)}$ forcing over $L[r]\left[g_{\xi}\right]$, for a name $\dot{g}_{\xi+1}$ for the generic filter for $\mathcal{P}_{<\gamma_{\xi+1}}$ which is the union of $g_{\xi}$ and of the generic filter for $\mathcal{P}_{<\gamma_{\xi}}$, that $\dot{g}_{\xi+1} \cap \mathcal{P}_{<\gamma_{\xi}}$ is $g_{\xi}$, that $\gamma_{\xi}$ is in $j_{g_{0}}, \dot{g}_{\xi+1}\left(\left\langle s_{\beta}: \beta<\gamma_{0}\right\rangle\right)\left(\alpha_{0}\right)$, and that $\gamma_{\xi}$ is in $j_{g_{\alpha}, \dot{g}_{\xi+1}}\left(d_{n}^{\alpha}\right)$. This shows that $g_{\xi+1}$ will have been chosen in such a way that $\gamma_{\xi} \in j_{g_{\alpha}, g_{\xi+1}}\left(d_{n}^{\alpha}\right)$. But $j_{g_{\alpha}, g_{\xi+1}}\left(d_{n}^{\alpha}\right)$ is contained in $S^{*}$. Since $S_{\alpha_{0}}^{*} \cap S^{*} \cap\left\{\gamma_{\xi}: \xi<\omega_{1}\right\}$ is unbounded in $\omega_{1}$, this shows that $T_{n}^{\alpha}$ is almost contained, modulo $\mathrm{NS}_{\omega_{1}}$, in $S^{*}$. Now, $T_{n}^{\alpha} \backslash S$ was chosen to be stationary, so that $S \triangle S^{*}$ is stationary after all. This contradiction finishes the proof.

Given a real $x$, the existence of $x^{\sharp}$ is equivalent to the fact that for every $X \subseteq \omega_{1}$ in $L[x]$ there is a club $C \subseteq \omega_{1}$ such that either $C \subseteq X$ or else $X \cap C=\emptyset$; in other words, the nonexistence of $x^{\sharp}$ is equivalent to the fact that some stationary and co-stationary subset of $\omega_{1}$ is constructible from $x$. Hence, the hypothesis used in Theorems 2.1 and 2.2 is optimal:

COROLlary 2.1. The following conditions are equivalent:

(1) $x^{\sharp}$ exists for every real $x$.

(2) Given any stationary and co-stationary $S \subseteq \omega_{1}$, any real $r$ and any sequence $\left\langle S_{\alpha}: \alpha<\omega_{1}\right\rangle$ of pairwise disjoint subsets of $\omega_{1}$ there are sets $S_{\alpha}^{*}\left(\alpha<\omega_{1}\right)$ such that each $S_{\alpha}$ is almost equal to $S_{\alpha}^{*}$ modulo $\mathrm{NS}_{\omega_{1}}$ and such that $S \notin L\left[r,\left\langle S_{\alpha}^{*}: \alpha<\omega_{1}\right\rangle\right]$.

(3) Given any real $r$, any sequence $\left\langle S_{\alpha}: \alpha<\omega_{1}\right\rangle$ of pairwise disjoint subsets of $\omega_{1}$, and any $S \subseteq \omega_{1}$ such that $S \cap S_{\alpha_{0}}$ and $S_{\alpha_{0}} \backslash S$ are stationary for some $\alpha_{0}$ there are sets $S_{\alpha}^{*}\left(\alpha<\omega_{1}\right)$ such that each $S_{\alpha}$ is almost equal to $S_{\alpha}^{*}$ modulo $\mathrm{NS}_{\omega_{1}}$ and such that $S \triangle S^{*}$ is stationary for every $S^{*} \in L\left[r,\left\langle S_{\alpha}^{*}: \alpha<\omega_{1}\right\rangle\right]$. 
There are models of ZFC in which some subset $A$ of $\omega_{1}$ admits, in a nontrivial way, a robust code with respect to some sequence $\left\langle S_{\alpha}: \alpha<\omega_{1}\right\rangle$ of pairwise disjoint stationary sets (in other words, $A$ is not constructible from any real and yet there is a real which codes $A$ in a robust way with respect to some sequence of pairwise disjoint stationary sets): Fix, in $L$, two collections $\mathcal{S}$ and $\mathcal{T}$ of size $\aleph_{1}$ of stationary subsets of $\omega_{1}$ such that all distinct members of $\mathcal{S} \cup \mathcal{T}$ are disjoint. Consider any extension over $L$ by any ccc forcing iteration $\mathcal{P}$ such that the final generic object can be coded by a subset $A$ of $\omega_{1}$ and such that every real in $L^{\mathcal{P}}$ appears at some initial stage of the iteration. In $L^{\mathcal{P}}$ build $\left\langle S_{\alpha}: \alpha<\omega_{1}\right\rangle$ by picking each $S_{\alpha}$ in $\mathcal{S}$ or in $\mathcal{T}$, and in such a way that the map from $\omega_{1}$ to $\{\mathcal{S}, \mathcal{T}\}$ sending each $\alpha$ to the collection to which $S_{\alpha}$ belongs codes $A$. It is clear that no real in $L^{\mathcal{P}}$ constructs $A$. Further, if $\left\langle S_{\alpha}^{*}: \alpha<\omega_{1}\right\rangle \in L^{\mathcal{P}}$ is such that each $S_{\alpha} \triangle S_{\alpha}^{*}$ is nonstationary in $L^{\mathcal{P}}$, then for every $\alpha$ there is a unique member $S$ of $\mathcal{S} \cup \mathcal{T}$ such that $S_{\alpha}^{*} \cap S$ is stationary in $L\left[\left\langle S_{\alpha}^{*}: \alpha<\omega_{1}\right\rangle\right]$, and this set $S$ is $S_{\alpha}$ (since, for any $S^{\prime} \in \mathcal{S} \cup \mathcal{T}$ different from $S_{\alpha}$, every club in $L^{\mathcal{P}}$ witnessing that the intersection of $S_{\alpha}^{*}$ with $S^{\prime}$ is nonstationary contains a club in $L$ ). It follows that $A$ is constructible from $\left\langle S_{\alpha}^{*}: \alpha<\omega_{1}\right\rangle$.

This construction can be easily modified in such a way that the coding of the set $A$ becomes nontrivial, in the sense that the real $r$ playing the role of code is necessarily nonconstructible: Let $\mathcal{P}$ be the standard forcing for adding $\aleph_{1}$ Cohen reals viewed as the partial order of finite functions contained in $\left(\omega_{1} \times \omega\right) \times\{0,1\}$. In $V^{\mathcal{P}}$ let $A$ code the generic filter $G$ and, letting $\vec{c}:=(\bigcup G)\left\lceil\left(\omega_{1} \backslash\{0\} \times \omega\right)\right.$, pick the sequence $\left\langle S_{\alpha}: \alpha<\omega_{1}\right\rangle$ in $L[\vec{c}]$ coding $\vec{c}$ in the same way as in the previous paragraph. Then, by the same argument as before, $c:=(\bigcup G)\lceil\{0\} \times \omega$ is a robust code for $A$ with respect to $\left\langle S_{\alpha}: \alpha<\omega_{1}\right\rangle$ and, as $c$ is Cohen generic over $L[\vec{c}], A \notin L\left[\left\langle S_{\alpha}: \alpha<\omega_{1}\right\rangle\right]$.

3. Restricting a decoding device to a club. One may ask whether the conclusion of Corollary 2.1(2) can be strengthened to yield the statement resulting from adding to the requirement that every $S_{\alpha} \triangle S_{\alpha}^{*}$ be nonstationary the extra requirement that each $S_{\alpha}^{*}$ be in fact completely contained in $S_{\alpha}$ or even the stronger requirement that, for some club $C \subseteq \omega_{1}, S_{\alpha}^{*}=S_{\alpha} \cap C$ for all $\alpha$. These questions can be best addressed after introducing a certain distinction between sequences of subsets of $\omega_{1}$. We say that a sequence $\left\langle S_{\alpha}: \alpha<\omega_{1}\right\rangle$ of subsets of $\omega_{1}$ is regressive if $S_{\alpha} \cap(\alpha+1)$ is empty for all $\alpha>0\left({ }^{13}\right)$, and otherwise we say that it is nonregressive $\left({ }^{14}\right)$.

$\left({ }^{13}\right)$ In other words, if the function sending each nonzero $\nu<\omega_{1}$ to the unique (if any) $\alpha$ such that $\nu \in S_{\alpha}$ is regressive on its domain.

$\left({ }^{14}\right)$ Note that the first manipulations in the given sequences of stationary sets in the proofs of Theorems 2.1 and 2.2 yield regressive sequences of stationary sets. 
Given any sequence of subsets of $\omega_{1}$ we can obviously shrink its members by countable segments so as to obtain a regressive sequence, so that the answer to the first of the above questions reduces to considering only regressive sequences. Concerning the second question, the following observation and Corollary 3.1 below show that regressive and nonregressive sequences may behave differently as to the existence of robust codes - in the present sense- for a given subset of $\omega_{1}$.

ObSeRvation 3.1. Given any subset $A$ of $\omega_{1}$ there is a nonregressive sequence $\left\langle S_{\alpha}: \alpha<\omega_{1}\right\rangle$ of pairwise disjoint stationary subsets of $\omega_{1}$ such that $A \in L\left[\left\langle S_{\alpha} \cap C: \alpha<\omega_{1}\right\rangle\right]$ for every club $C \subseteq \omega_{1}$.

Proof. Let $\mathcal{S}=\left\langle\bar{S}_{\xi}: \xi<\omega_{1}\right\rangle$ and $\left\langle T_{\alpha}: \alpha<\omega_{1}\right\rangle$ be sequences of mutually disjoint stationary subsets of $\omega_{1}$ such that $\mathcal{S}$ is regressive and $S_{\alpha} \cap T_{\beta}=\emptyset$ for all $\alpha, \beta<\omega_{1}$, and let $\left\langle X_{\alpha}: \alpha<\omega_{1}\right\rangle$ be a constructible partition of $\omega_{1}$ into unbounded sets. Fix also, for every $\alpha$, a one-to-one map $f_{\alpha}: T_{\alpha} \rightarrow X_{\alpha}$ such that $f_{\alpha}(\nu)>\nu$ for all $\nu \in T_{\alpha}$. We build a third sequence $\left\langle S_{\alpha}: \alpha<\omega_{1}\right\rangle$ of pairwise disjoint stationary sets by setting $S_{f_{\alpha}(\nu)}=\{\nu\} \cup$ $\bar{S}_{f_{\alpha}(\nu)}$ for each $\alpha \in A$ and each $\nu \in T_{\alpha}$, and by setting $S_{\xi}=\bar{S}_{\xi}$ for all $\xi \in \omega_{1} \backslash \bigcup_{\alpha \in A}$ range $\left(f_{\alpha}\right)$. By construction, $\left\langle S_{\alpha}: \alpha<\omega_{1}\right\rangle$ consists of pairwise disjoint stationary sets and, since all $T_{\alpha}$ 's are stationary, it is easy to see that, given any club $C \subseteq \omega_{1}, A$ is the set of $\alpha$ 's for which there is some nonzero $\xi \in X_{\alpha}$ such that $\overline{S_{\xi}} \cap C \cap(\xi+1)$ is nonempty. Hence, $A \in L\left[\left\langle S_{\alpha} \cap C: \alpha<\omega_{1}\right\rangle\right]$ for every club $C \subseteq \omega_{1}$.

I do not see how to adapt the present proof of Theorem 2.1 in order to deal with the second question above, for regressive sequences of stationary subsets of $\omega_{1}$, in general (under the assumption that the sharp of every real exists). Nevertheless, as Corollary 3.1 shows, the corresponding form of Corollary 2.1(2) does hold, under $\mathrm{AD}^{L(\mathbb{R})}$, in any $\mathbb{P}_{\max }$ extension of $L(\mathbb{R})\left({ }^{15}\right)$.

In an earlier version of this paper I proved Corollary 3.1, via the absoluteness Theorem 4.65 from [W], from the stronger assumption that AD holds in $L(\mathbb{R})$ and that there is a Woodin cardinal with a measurable cardinal above. Using the essence of the proof of the old version of (the first part of) Corollary 3.1 together with standard facts of the $\mathbb{P}_{\max }$ theory under $\mathrm{AD}^{L(\mathbb{R})}$, an anonymous referee found a simplified argument for proving that result from just $\mathrm{AD}^{L(\mathbb{R})}$. The proof of the more general Theorem 3.1, or at least the proof of its first part, arises quite naturally out of the referee's argument.

$\left({ }^{15}\right)$ Remember that, under $\mathrm{AD}^{L(\mathbb{R})}$, any $\mathbb{P}_{\max }$ extension of $L(\mathbb{R})$ is a model of ZFC in which $\mathrm{AD}^{L(\mathbb{R})}$ holds. 
Theorem 3.1. Under $\mathrm{AD}^{L(\mathbb{R})}$, given any formula $\varphi(x, y)$ of set theory with parameters in $L(\mathbb{R})$ and any $\mathbb{P}_{\max }$ generic filter $G$ over $L(\mathbb{R})$, the following statement about $\varphi(x, y)$ holds in $L(\mathbb{R})[G]$ :

Suppose there is a stationary and co-stationary $S \subseteq \omega_{1}$ and there is a regressive sequence $\mathcal{S}=\left\langle S_{\alpha}: \alpha<\omega_{1}\right\rangle$ of pairwise disjoint stationary subsets of $\omega_{1}$ such that $\varphi\left(S, \mathcal{S}\lceil C)\right.$ holds for every club $C \subseteq \omega_{1}$, where $\mathcal{S}\lceil C$ denotes the sequence $\left\langle S_{\alpha} \cap C: \alpha<\omega_{1}\right\rangle$. Then there is a subset $\mathcal{X}$ of $\mathcal{P}\left(\omega_{1}\right)$ of size $2^{\aleph_{1}}$ and there is a club $C \subseteq \omega_{1}$ such that $\varphi(A, \mathcal{S}\lceil C)$ holds for all $A \in \mathcal{X}$. Moreover, if there is some $\alpha_{0}$ for which both $S \cap S_{\alpha_{0}}$ and $S_{\alpha_{0}} \backslash S$ are stationary, then $\mathcal{X}$ can be taken so that $A \triangle B$ is stationary for all distinct $A$ and $B$ in $\mathcal{X}$.

Corollary 3.1. Under the $\mathbb{P}_{\max }$ axiom $(*)$, for every stationary and co-stationary $S \subseteq \omega_{1}$, every regressive sequence $\left\langle S_{\alpha}: \alpha<\omega_{1}\right\rangle$ of pairwise disjoint stationary subsets of $\omega_{1}$ and every real $r$ there is a club $C \subseteq \omega_{1}$ such that $S \notin L\left[r,\left\langle S_{\alpha} \cap C: \alpha<\omega_{1}\right\rangle\right]$. Furthermore, if there is some $\alpha_{0}$ such that $S \cap S_{\alpha_{0}}$ and $S_{\alpha_{0}} \backslash S$ are stationary, then there is a club $C \subseteq \omega_{1}$ with the property that $S \triangle S^{\prime}$ is stationary for every subset $S^{\prime}$ of $\omega_{1}$ in $L\left[r,\left\langle S_{\alpha} \cap C: \alpha<\omega_{1}\right\rangle\right]$.

Proof. By taking $\varphi(x, y)$ in Theorem 3.1 to be $x \in L[r, y]$ it follows that, under $(*)$, there cannot be any stationary and co-stationary $S \subseteq \omega_{1}$ and any regressive sequence $\left\langle S_{\alpha}: \alpha<\omega_{1}\right\rangle$ of mutually disjoint stationary subsets of $\omega_{1}$ such that $S \in L\left[r,\left\langle S_{\alpha} \cap C: \alpha<\omega_{1}\right\rangle\right]$ for every club $C \subseteq \omega_{1}$. The reason for this is that otherwise, by the conclusion of the theorem, $\mathcal{P}\left(\omega_{1}\right)^{L\left[r,\left\langle S_{\alpha} \cap C: \alpha<\omega_{1}\right\rangle\right]}$ would be of size $2^{\aleph_{1}}$ for some club $C \subseteq \omega_{1}$. However, under $(*)$ the sharp of every subset $A$ of $\omega_{1}$ exists (and therefore in $L[A]$ there are only $\aleph_{1}$ subsets of $\left.\omega_{1}\right)$.

For the second conclusion let $\varphi(x, y)$ be a formula with parameter $r$ saying that there is some $x^{\prime} \subseteq \omega_{1}$ in $L[r, y]$ with $x \triangle x^{\prime}$ nonstationary. Assume $(*)$ and suppose the conclusion fails. Let $\left\langle S_{\alpha}: \alpha<\omega_{1}\right\rangle$ be a regressive sequence of mutually disjoint stationary subsets of $\omega_{1}$, let $\alpha_{0}$ be a countable ordinal and let $S \subseteq \omega_{1}$ be such that $S \cap S_{\alpha_{0}}$ and $S_{\alpha_{0}} \backslash S$ are stationary, and suppose $H_{\omega_{2}} \models \varphi\left(S,\left\langle S_{\alpha} \cap C: \alpha<\omega_{1}\right\rangle\right)$ holds for every club $C$. Then there is a club $C \subseteq \omega_{1}$ and there is a set $\mathcal{X}$ of size $2^{\aleph_{1}}$ of subsets of $\omega_{1}$ such that $A \triangle B$ is stationary for all distinct $A, B \in \mathcal{X}$ and such that, for every given $A \in \mathcal{X}$, there is some $A^{\prime} \subseteq \omega_{1}$ with $A \triangle A^{\prime}$ nonstationary and $A^{\prime} \in$ $L\left[r,\left\langle S_{\alpha} \cap C: \alpha<\omega_{1}\right\rangle\right]$. But the map sending $A \in \mathcal{X}$ to $A^{\prime}$ is then one-to-one, so that $\mathcal{P}\left(\omega_{1}\right)^{L\left[r,\left\langle S_{\alpha} \cap C: \alpha<\omega_{1}\right\rangle\right]}$ is of size $2^{\aleph_{1}}$, which again is a contradiction.

Note that the conclusion of Corollary 3.1 cannot be strengthened to say that for every real $r$, every stationary and co-stationary $S \subseteq \omega_{1}$ and every regressive sequence $\left\langle S_{\alpha}: \alpha<\omega_{1}\right\rangle$ of mutually disjoint stationary subsets of $\omega_{1}$ there is a club $C \subseteq \omega_{1}$ such that $S \notin L\left[r,\left\langle S_{\alpha} \cap D: \alpha<\omega_{1}\right\rangle\right]$ for 
every club $D \subseteq C$. The reason is that, given any $A \subseteq \omega_{1}$ and any sequence $\left\langle S_{\alpha}: \alpha<\omega_{1}\right\rangle$ as above, one can always find a club $D \subseteq \omega_{1}$ such that $\left\langle S_{\alpha} \cap D: \alpha<\omega_{1}\right\rangle$ codes $A$ : one may pick $D$ for example in such a way that, for each $\alpha<\omega_{1}$, the $\alpha$ th nonlimit point of $\bigcup_{\xi<\omega_{1}} S_{\xi} \cap D$ is in $S_{\alpha}$ if and only if $\alpha$ is in $A$.

Note also that the conclusion of Theorem 3.1 does not hold if we replace regressive sequences of mutually disjoint stationary sets by regressive functions from $\omega_{1}$ into $\omega_{1}$ : Given any function $\mathcal{F} \subseteq \omega_{1} \times \omega_{1}$ there is exactly one set $A \subseteq \omega_{1}$ such that, given any club $C \subseteq \omega_{1}, A=\left\{\alpha<\omega_{1}\right.$ : $\left(\mathcal{F}\lceil C)^{-1}(\alpha)\right.$ is stationary $\}$.

Proof of Theorem 3.1. Fix a formula $\varphi(x, y)$ with parameters in $L(\mathbb{R})$ for which $\mathbb{P}_{\max }$ forces the antecedent of the statement about $\varphi(x, y)$ expressed in the theorem $\left({ }^{16}\right)$. By changing $\varphi(x, y)$ slightly if necessary we may assume that the sequence $\mathcal{S}$ witnessing the above hypothesis is forced to define a partition of $\omega_{1}$.

By [W, Theorem 4.50], there is a $\mathbb{P}_{\max }$ condition $\langle(M, I), a\rangle$ and there are an $I$-positive $s \in M$ such that $\omega_{1} \backslash s$ is also $I$-positive and a regressive partition $\left\langle s_{i}: i\left\langle\omega_{1}^{M}\right\rangle \in M\right.$ of $\omega_{1}^{M}$ into $I$-positive sets such that $\langle(M, I), a\rangle$ forces that, letting $j_{\dot{G}}:(M, I) \rightarrow\left(M^{*}, I^{*}\right)$ be the unique iteration of $(M, I)$ sending $a$ to $A_{\dot{G}}\left({ }^{17}\right)$, (where $\dot{G}$ is a term for the generic filter) and letting $\mathcal{S}_{\dot{G}}$ be $j_{\dot{G}}\left(\left\langle s_{i}: i<\omega_{1}^{M}\right\rangle\right), \varphi\left(j_{\dot{G}}(s), \mathcal{S}_{\dot{G}}\lceil C)\right.$ holds for all clubs $C \subseteq \omega_{1}$.

Let $x$ be a real coding $(M, I)$. Let $G$ be generic for $\mathbb{P}_{\max }$ over $L(\mathbb{R})$ containing $\langle(M, I), a\rangle$. In order to finish the proof (of the theorem without its 'Moreover' part) it suffices to show that for every function $F: \omega_{1} \rightarrow \omega_{1}$ in $L(\mathbb{R})[G]$ there is, in $L(\mathbb{R})[G]$, some $A \subseteq \omega_{1}$ such that, for all $\nu<\omega_{1}$, the $\nu$ th successor Silver indiscernible for $L[x]$ is in $A$ if and only if $F(\nu)=1$ and such that $\varphi\left(A, \mathcal{S}_{G}\lceil C)\right.$ holds, where $C$ is the club of limit Silver indiscernibles for $L[x]$ below $\omega_{1}$.

Let $\langle(N, J), b\rangle$ be any condition in $G$ extending $\langle(M, I), a\rangle$. Again by [W, Theorem 4.50] we may assume, towards a contradiction, that there is a function $f: \omega_{1}^{N} \rightarrow \omega_{1}^{N}$ in $N$ such that $\langle(N, J), b\rangle$ forces that, letting $\mathcal{X}$ be the set of $A \subseteq \omega_{1}$ such that, for all $\nu$, the $\nu$ th successor indiscernible for $L[x]$ is in $A$ if and only if $j_{\dot{G}}(f)(\nu)=1$, there is no $A$ in $\mathcal{X}$ such that $\varphi\left(A, \mathcal{S}_{\dot{G}}\lceil C)\right.$.

Since every Silver indiscernible for $L[x]$ is in the critical sequence of every iteration of $(M, I)$, in $N$ we can construct an iteration $k:(M, I) \rightarrow\left(M^{*}, I^{*}\right)$ of length $\omega_{1}^{N}$ such that

(1) $\mathcal{P}^{M^{*}}\left(\omega_{1}^{N}\right) \cap J=I^{*}$

$\left({ }^{16}\right)$ By homogeneity of $\mathbb{P}_{\max }$ under $\mathrm{AD}^{L(\mathbb{R})}$, if some condition forces that hypothesis about $\varphi$, then so does every condition.

$\left({ }^{17}\right)$ Given a filter $G$ of $\mathbb{P}_{\max }, A_{G}$ will denote the union of all $b$ such that $\langle(N, J), b\rangle \in G$ for some $N$ and $J$. 
(2) for all $\xi, \nu<\omega_{1}^{N}$, if $\xi$ is the $\nu$ th successor Silver indiscernible for $L[x]$, then $\xi \in k(s)$ if and only if $f(\nu)=1$,

(3) letting $j$ be the unique iteration of $(M, I)$ sending $a$ to $b$, for every limit Silver indiscernible $\xi<\omega_{1}^{N}$ for $L[x]$ and every $\gamma<\omega_{1}^{N}, \xi \in$ $k\left(\left\langle s_{i}: \xi<\omega_{1}^{M}\right\rangle\right)(\gamma)$ if and only if $\xi \in j\left(\left\langle s_{i}: \xi<\omega_{1}^{M}\right\rangle\right)(\gamma)$.

The above construction can be carried out with the help of a bookkeeping argument very much as in [W, Lemma 4.36]: We start fixing a partition $\left\langle B_{\alpha, \beta, \gamma}: \alpha, \beta, \gamma<\omega_{1}^{N}\right\rangle$ in $N$ consisting of $J$-positive sets such that, for all $\alpha, \beta$ and $\gamma, B_{\alpha, \beta, \gamma} \cap(\alpha+1)=\emptyset$ and such that, for all $\alpha$ and $\beta, B_{\alpha, \beta, \gamma}$ is contained in $j\left(\left\langle s_{i}: i<\omega_{1}^{M}\right\rangle\right)(\gamma)$. The iteration $k$ will be of the form $\left\langle\left(M_{\alpha}, I_{\alpha}\right), G_{\alpha^{\prime}}, k_{\alpha^{\prime}, \alpha}: \alpha^{\prime}<\alpha<\omega_{1}^{N}\right\rangle$. Given some $\alpha$ for which $\left(M_{\alpha}, I_{\alpha}\right)$ has already been defined, we fix a sequence $\left\langle X_{\beta, \gamma}^{\alpha}: \beta, \gamma<\omega_{1}^{M_{\alpha}}\right\rangle$ such that, given any $\gamma<\omega_{1}^{M_{\alpha}},\left\langle X_{\beta, \gamma}^{\alpha}: \beta<\omega_{1}^{M}\right\rangle$ enumerates all $I_{\alpha}$-positive subsets of $k_{0, \alpha}\left(\left\langle s_{i}: i<\omega_{1}^{M}\right\rangle\right)(\gamma)$ in $M_{\alpha}$. Then, given any Silver indiscernible $\xi$ for $L[x]$,

(a) if $\xi$ is the $\nu$ th successor indiscernible, we put $k_{0, \xi}(s)$ in $G_{\xi}$ if and only if $j(f)(\nu)=1$,

(b) if $\xi$ is a limit indiscernible and $\xi$ is in $B_{\alpha, \beta, \gamma}$, then we put $k_{\alpha, \xi}\left(X_{\beta, \gamma}^{\alpha}\right)$ in $G_{\xi}$.

Now let $H$ be, in $L(\mathbb{R})[G]$, the set of all $\mathbb{P}_{\max }$ conditions $\left\langle\left(M^{\prime}, I^{\prime}\right), a^{\prime}\right\rangle$ for which there is a (unique) iteration $j^{\prime}:\left(M^{\prime}, I^{\prime}\right) \rightarrow\left(M^{\prime \prime}, I^{\prime \prime}\right)$ of $\left(M^{\prime}, I^{\prime}\right)$ sending $a^{\prime}$ to $j_{G}(k(a))$ and such that $I^{\prime \prime}=\mathcal{P}\left(\omega_{1}\right)^{M^{\prime \prime}} \cap \mathrm{NS}_{\omega_{1}}$. By the proof of Theorem 4.60 in [W] and by Lemma 4.56 in [W], $H$ is $\mathbb{P}_{\max }$ generic over $L(\mathbb{R})$ and $L(\mathbb{R})[G]=L(\mathbb{R})[H]$. Moreover, $H$ contains $\langle(N, J), k(a)\rangle$, which, by the construction of $k$, is a condition extending $\langle(M, I), a\rangle$ as witnessed by $k$, and thus forcing that $\varphi\left(j_{\dot{G}}(k(s)),\left\langle j_{\dot{G}}\left(\left\langle s_{i}: i<\omega_{1}^{M}\right\rangle\right)(\alpha) \cap D: \alpha<\omega_{1}\right\rangle\right)$ holds for every club $D$ of $\omega_{1}$, and in particular for $C$. Note that $j_{H}\left\lceil N\right.$ and $j_{G}\lceil N$ send $k(a)$ to the same set $j_{G}(k(a))$, and therefore $j_{G}:(N, J) \rightarrow\left(N^{\prime}, J^{\prime}\right)$ and $j_{H}:(N, J) \rightarrow\left(N^{\prime}, J^{\prime}\right)$ are the same iteration of $(N, J)$. Finally, again by the construction of $k$,

(i) for every $\nu<\omega_{1}^{N}$, the $\nu$ th successor Silver indiscernible for $L[x]$ is in $k(s)$ if and only if $f(\nu)=1$, and therefore, for every $\nu<\omega_{1}$, the $\nu$ th successor Silver indiscernible for $L[x]$ is in $j_{H}(k(s))\left(=j_{G}(k(s))\right)$ if and only if $j_{G}(f)(\nu)=1$,

(ii) $j\left(\left\langle s_{i}: i<\omega_{1}^{M}\right\rangle\right)(\alpha) \cap C \cap \omega_{1}^{N}=k\left(\left\langle s_{i}: i<\omega_{1}^{M}\right\rangle\right)(\alpha) \cap C \cap \omega_{1}^{N}$ for every $\alpha<\omega_{1}^{N}$, and therefore $j_{G}\left(j\left(\left\langle s_{i}: i<\omega_{1}^{M}\right\rangle\right)\right)(\alpha) \cap C$ is equal to $j_{H}\left(k\left(\left\langle s_{i}: i<\omega_{1}^{M}\right\rangle\right)\right)(\alpha) \cap C\left(=j_{G}\left(k\left(\left\langle s_{i}: i<\omega_{1}^{M}\right\rangle\right)\right)(\alpha) \cap C\right)$ for every $\alpha<\omega_{1}$.

Hence, on the one hand we see that $j_{H}(k(s))$ is a member of $\mathcal{X}$, and on the other hand $\varphi\left(j_{H}(k(s)),\left\langle j_{G}\left(j\left(\left\langle s_{i}: i<\omega_{1}^{M}\right\rangle\right)\right)(\alpha) \cap C: \alpha<\omega_{1}\right\rangle\right)$ holds, which contradicts the choice of $\langle(N, J), b\rangle$ since $\langle(N, J), b\rangle$ is in $G$. 
Suppose now that $\mathbb{P}_{\max }$ forces the hypothesis about $\varphi(x, y)$ expressed in the 'Moreover' part of the theorem. Fix corresponding $\langle(M, I), a\rangle \in \mathbb{P}_{\max }$ and $s,\left\langle s_{i}: i\left\langle\omega_{1}^{M}\right\rangle \in M\right.$ as in the proof of the first part of the theorem. Fix also an ordinal $\alpha<\omega_{1}^{M}$ such that $s_{\alpha} \cap s$ and $s_{\alpha} \backslash s$ are both $I$-positive and again let $x$ be a real coding $\langle(M, I), a\rangle$. Let $\langle(N, J), b\rangle$ be a condition extending $\langle(M, I), a\rangle$ such that $x \in N$ and let $\left\langle t_{i}: i<\omega_{1}^{N}\right\rangle$ be a partition in $N$ of $j\left(s_{\alpha}\right)$ into $J$-positive sets, where $j$ is the iteration of $(M, I)$ sending $a$ to $b$. This time it will suffice to show that for every $\mathbb{P}_{\max }$ generic filter $G$ over $L(\mathbb{R})$ containing $\langle(N, J), b\rangle$ and every proper subset $B$ of $\omega_{1}$ in $L(\mathbb{R})[G]$ there is, in $L(\mathbb{R})[G]$, some $A \subseteq \omega_{1}$ such that

(a) $j_{G}\left(\left\langle t_{i}: i<\omega_{1}^{N}\right\rangle\right)(\beta) \backslash A$ is nonstationary for all $\beta \in B$ and $j_{G}\left(\left\langle t_{i}\right.\right.$ : $\left.\left.i<\omega_{1}^{N}\right\rangle\right)(\beta) \cap A$ is nonstationary for all $\beta \in \omega_{1} \backslash B$,

(b) $\varphi\left(A, \mathcal{S}_{G}\lceil C)\right.$ holds, where $C$ is the club of countable Silver indiscernibles for $L[x]$.

Fix therefore a condition $\left\langle\left(N^{\prime}, J^{\prime}\right), b^{\prime}\right\rangle \in G$ extending $\langle(N, J), b\rangle$ and a proper subset $\bar{b}$ of $\omega_{1}^{N^{\prime}}$ in $N^{\prime}$ and assume, towards a final contradiction, that $\left\langle\left(N^{\prime}, J^{\prime}\right), b^{\prime}\right\rangle$ forces that there is no $A \subseteq \omega_{1}$ such that (a) and (b) above (with $\dot{G}$ instead of $G$ and $j_{\dot{G}}(\bar{b})$ instead of $B$ ) hold. In $N^{\prime}$ there is then an iteration $k:(M, I) \rightarrow\left(M^{*}, I^{*}\right)$ of $(M, I)$ such that, letting $j_{0}$ be the iteration of $(M, I)$ sending $a$ to $b^{\prime}$ and letting $j_{1}$ be the iteration of $(N, J)$ sending $b$ to $b^{\prime}$,

(1) $\mathcal{P}\left(\omega_{1}^{N^{\prime}}\right)^{M^{*}} \cap J^{\prime}=I^{*}$,

(2) given any Silver indiscernible $\xi<\omega_{1}^{N^{\prime}}$ for $L[x]$, if $\gamma<\omega_{1}^{N^{\prime}}$ is such that $\xi \in j_{1}\left(\left\langle t_{i}: i<\omega_{1}^{N}\right\rangle\right)(\gamma)$, then $\xi \in k(s)$ if and only if $\gamma \in \bar{b}$,

(3) for every Silver indiscernible $\xi<\omega_{1}^{N^{\prime}}$ for $L[x]$ and every $\gamma<\omega_{1}^{N^{\prime}}$, $\xi \in k\left(\left\langle s_{i}: i<\omega_{1}^{M}\right\rangle\right)(\gamma)$ if and only if $\xi \in j_{0}\left(\left\langle s_{i}: i<\omega_{1}^{M}\right\rangle\right)(\gamma)$.

Such an iteration $k$ can be easily constructed in $N^{\prime}$ using a bookkeeping argument as in the first part of the proof. We are able to make (2) work because $s_{\alpha} \cap s$ and $s_{\alpha} \backslash s$ are both $I$-positive. The rest of the argument is now as in the first part of the theorem: We let $H$ be the set of all $\mathbb{P}_{\max }$ conditions $\left\langle\left(M^{\prime}, I^{\prime}\right), a^{\prime}\right\rangle$ for which there is a (unique) iteration $j^{\prime}:\left(M^{\prime}, I^{\prime}\right) \rightarrow\left(M^{\prime \prime}, I^{\prime \prime}\right)$ of $\left(M^{\prime}, I^{\prime}\right)$ sending $a^{\prime}$ to $j_{G}(k(a))$ and such that $I^{\prime \prime}=\mathcal{P}\left(\omega_{1}\right)^{M^{\prime \prime}} \cap \mathrm{NS}_{\omega_{1}}$. Again, $H$ is $\mathbb{P}_{\max }$ generic over $L(\mathbb{R}), L(\mathbb{R})[G]=$ $L(\mathbb{R})[H]$, and $H$ contains $\langle(N, J), k(a)\rangle$, which is a condition extending $\langle(M, I), a\rangle$ as witnessed by $k$. Hence, on the one hand $\varphi\left(j_{H}(k(s))\right.$, $\left.\left\langle j_{H}\left(k\left(\left\langle s_{i}: i<\omega_{1}^{M}\right\rangle\right)\right)(\alpha) \cap C: \alpha<\omega_{1}\right\rangle\right)$ holds-and therefore, by $(3)$ of the construction, so does $\varphi\left(j_{H}(k(s)),\left\langle j_{G}\left(\left\langle s_{i}: i<\omega_{1}^{M}\right\rangle\right)(\alpha) \cap C\right.\right.$ : $\left.\left.\alpha<\omega_{1}\right\rangle\right)$-and on the other hand $j_{H}(k(s))$ has the property that $j_{G}\left(\left\langle t_{i}: i<\omega_{1}^{N}\right\rangle\right)(\beta) \backslash j_{H}(k(s))$ is nonstationary for all $\beta \in j_{G}(\bar{b})$ and $j_{G}\left(\left\langle t_{i}: i<\omega_{1}^{N}\right\rangle\right)(\beta) \cap j_{H}(k(s))$ is nonstationary for all $\beta \in \omega_{1} \backslash j_{G}(\bar{b})\left({ }^{18}\right)$.

$\left({ }^{18}\right)$ Because $j_{G}(x)=j_{H}(x)$ for all $x \in N^{\prime}$. 
But that is a contradiction to the choice of $\left\langle\left(N^{\prime}, J^{\prime}\right), b^{\prime}\right\rangle$ since $\left\langle\left(N^{\prime}, J^{\prime}\right), b^{\prime}\right\rangle$ is in $G$.

Every function with domain $\omega_{1}$ and finite sequences of countable ordinals as values can obviously be coded by a function from $\omega_{1}$ into $\omega_{1}$, so that the simplest decoding devices - from the point of view of the information they carry - that one may consider beyond the functions from $\omega_{1}$ into $\omega_{1}$ are the functions from $\omega_{1}$ into the set of countable subsets of $\omega_{1}$. I will finish the paper with a simple observation concerning decoding devices $\mathcal{F}$ of this last kind, which shows the existence, under $\mathrm{MA}_{\omega_{1}}$, of codes $r \in \mathbb{R}$ having the stronger kind of robustness that ensures that the coding of a given set be realizable also in the presence of every function $\mathcal{F}^{*} \subseteq \mathcal{F}$ such that the domain of $\mathcal{F}^{*}$ is merely an unbounded subset of the domain of $\mathcal{F}$.

ObSeRvation 3.2. $\left(\mathrm{MA}_{\omega_{1}}\right)$ Given any set $X$ of size $\aleph_{1}$ of members of the Baire space and any $A \subseteq \omega_{1}$ there is a real $r$ such that $A \in L[r, Y]$ whenever $Y$ is an uncountable subset of $X$.

Proof. Let $\left\langle r_{\alpha}: \alpha<\omega_{1}\right\rangle$ be a one-to-one enumeration of $X$. Fix a recursive list $\left\langle\sigma_{n}: n<\omega\right\rangle$ of the finite sequences of integers and also a recursive partition $\left\langle e_{i}: i<\omega\right\rangle$ of $\omega$ into infinite sets. Define $\left\langle a_{i}: i<\omega\right\rangle$ by letting $a_{i}$ be, for each $i$, the set of $n<\omega$ such that the length of $\sigma_{n}$ is in $e_{i}$. Then we define sets of integers $a_{i}^{\alpha}\left(i<\omega, \alpha<\omega_{1}\right)$ by letting $a_{i}^{\alpha}$ be the set of $n \in a_{i}$ such that $\sigma_{n}$ is an initial segment of $r_{\alpha}$. Note that, for every $i<\omega$ and for all distinct $\alpha, \beta<\omega_{1}, a_{i}^{\alpha}$ and $a_{i}^{\beta}$ are almost disjoint subsets of $a_{i}$ and that each $r_{\alpha}$ is recursively equivalent to $\left(a_{i}^{\alpha}: i<\omega\right)$. Let $A_{\alpha}$ be, for each $\alpha<\omega_{1}$, a set of integers coding $A \cap \alpha$. For each $i$ we can find, by $\mathrm{MA}_{\omega_{1}}, x_{i} \subseteq \omega$ such that $x_{i} \cap a_{i}^{\alpha}$ is infinite exactly when $i \in A_{\alpha}$. Letting now $x$ be a real coding $\left(x_{i}\right)_{i}$, we deduce that $A_{\alpha}$, and therefore also $A \cap \alpha$, can be decoded from $x$ and $r_{\alpha}$ for each $\alpha$. Now the desired result follows trivially since, given any uncountable $Y \subseteq X, A$ is the union of the sets decoded by $x$ together with some real in $Y$.

\section{References}

[J-So] R. Jensen and R. Solovay, Some applications of almost disjoint sets, in: Mathematical Logic and Foundations of Set Theory (Jerusalem, 1968), Y. Bar-Hillel (ed.), North-Holland, Amsterdam, 1970, 84-104.

[L] P. Larson, Martin's Maximum and the $\mathbb{P}_{\max }$ axiom (*), Ann. Pure Appl. Logic 106 (2000), 135-149.

[M] J. Moore, Proper forcing, the continuum, and set mapping reflection, preprint, 2003.

[S] R. Schindler, Semi-proper forcing, remarkable cardinals, and Bounded Martin's Maximum, MLQ Math. Log. Q 50 (2004), 527-532. 
[T] S. Todorčević, Generic absoluteness and the continuum, Math. Res. Lett. 9 (2002), 465-471.

[W] H. Woodin, The Axiom of Determinacy, Forcing Axioms, and the Nonstationary Ideal, de Gruyter Ser. Logic Appl. 1, de Gruyter, Berlin, 1999.

Department of Mathematics

University of Bristol

University Walk

Bristol, BS8 1TW, England, UK

E-mail: David.Aspero@bristol.ac.uk

Received 17 June 2004;

in revised form 8 July 2005 